\title{
Monitoring and assessment of leaf litter dynamics in a mixed mangal forest of the Cross River estuary, Nigeria
}

\author{
Edu. Esther. Aja. B ${ }^{1}$, Nsirim. L. Edwin. Wosu ${ }^{2, ~ *, ~ M a r t i n s, ~ O . ~ O n o n y u m e ~}{ }^{1}$ \\ ${ }^{1}$ Department of Botany, University of Calabar, Calabar, Nigeria \\ ${ }^{2}$ Department of Plant Sciences and Biotechnology, University of Port Harcourt, Nigeria
}

Email address:

esiedu@yahoo.com (E. E. Aja. B), nsirim.edwin-wosu@uniport.edu.ng (N. L. E. Wosu)

\section{To cite this article:}

Edu. Esther. Aja. B, Nsirim. L. Edwin. Wosu, Martins, O. Ononyume. Monitoring and Assessment of Leaf Litter Dynamics in a Mixed Mangal Forest of the Cross River Estuary, Nigeria. International Journal of Environmental Monitoring and Analysis. Vol. 2, No. 3, 2014, pp. 163-174. doi: 10.11648/j.ijema.20140203.16

\begin{abstract}
The leaf litter decomposition of Nypa fruticans, Rhizophora racemosa and Avicennia africana were studied across a tidal gradient in a mixed mangrove forest of the Cross River estuary Nigeria. Leaf litter decomposition was measured along the tidal gradients (low, mid, high) using litter bags. A single exponential model was used to study the decomposition rates of the leaves. Leaf decomposition varied significantly $(\mathrm{P}<0.001)$ among species and spatially across tidal gradients over the study period. Decomposition was fastest in A. africana and slowest in $N$. fruticans and spatially, it was fastest at the low tide level and slowest at the high tide level. Tidal effects were much larger than species differences in the decomposition rates. The time (days) required for the loss of half the initial dry mass $\left(\mathrm{T}_{50}\right)$ of the decomposing leaves at the low, mid and high tide levels were A. africana, 46, 57 and 77, R. racemosa 69,86 and 115 , and $N$. fruticans 86,99 and 115 respectively. Estimates of leaf litter turnover rates showed that the actual litter turnover based on the relative measure of leaf litterfall and biomass on the forest floor $\left(\mathrm{K}_{\mathrm{t}}\right)$ were much shorter than the projected litter turnover based on the leaf decomposition rates $\left(\mathrm{K}_{\mathrm{d}}\right)$. The estimated actual residence times were less than one day while the projected residence times ranged from 83 to 142 days across tidal gradients, suggesting involvement of other ecological processes in litter loss and their possible transport into the Cross River estuary. The nitrogen contents and nutritional value of the decomposing leaves increased with time during the study. The increase varied significantly $(\mathrm{P}<0.001)$ among species, as well as temporally $(\mathrm{P}$ $<0.001)$ and spatially $(\mathrm{P} \leq 0.05)$. The average $\mathrm{C}: \mathrm{N}$ ratio decreased from 27.7 to 22.1 in A. africana, 26.4 to 23.9 in $R$. racemosa and 32.8 to 23.6 in $N$. fruticans. The overall changes in nutrients during decomposition indicated net mineralization. Mangrove leaf litter dynamics, trophic value and organic matter exchange of the system have implications on the productivity of the Cross River estuary and ultimately the Gulf of Guinea. Knowledge of these processes is critical for the maintenance and long term sustainability of the mangrove and surrounding ecosystems.
\end{abstract}

Keywords: Leaf Decomposition, Spatial Variation, Temporal Variation, Total Carbon, Total Nitrogen

\section{Introduction}

There is a gradual estimated worldwide loss of mangrove ecosystems at $1-2 \% \mathrm{y}^{-1}$ possibly as a result of high level ecological demand and unsustainable management practices [1]. Due to poor knowledge and understanding of the functions and services that mangroves provide, the importance of the system to the environment is undermined leading to undue exploitation and even extinction. Despite the worldwide clarion call for management of mangroves [2], degradation and outright destruction of mangroves have continued unabated in Nigeria, with policy-makers paying little or no attention.

Mangroves are halophytic trees that dominate the intertidal zone along coastlines, estuaries and islands in tropical and sub-tropical regions of the world where they exist under high marine environmental conditions [3, 4]. They form distinct communities called mangrove forests or mangals covering riverbanks, estuaries, sea coasts, as well as carbonate sands and coral rubble islands especially in the tropics and sub-tropics [5]. They are best developed in tropical estuaries which receive evenly distributed heavy 
rainfall throughout the year [6]. Mangroves are extremely important to the nutrient budgets of adjoining estuaries and other coastal waters because of their high productivity. Based on unabated ecological demand and unsustainable management practices, there is need for a better understanding of the system.

The assessment of productivity of a mangrove ecosystem requires an understanding of the key processes of production and decomposition of mangrove litter. Litter dynamics is a measure of productivity in an ecosystem. The high productivity of mangrove ecosystems has been partly attributed to high leaf production and litterfall and rapid decomposition of the litter [7]. Litter decomposition enhanced by sedimentary bacteria and fungi commences when fallen litter are subjected to breakdown process, leading to detritus derivation and subsequent release of nutrients. The formation of detritus and nutrient release directly increase the benthic primary production and contribute to estuarine secondary production on a large scale [8]. Mangrove litter in estuaries has been well documented as an important base in the maintenance of detrital based food web which is important in the production of commercial fishes and invertebrates [9]. The energy transfer through mangrove litters as a result of detritus loading and export leads to the evolution of rich biodiversity resources in mangrove ecosystems [10, 11]. This high productivity turnover rate of organic matter and the exchange with terrestrial and marine ecosystems make mangroves so important in the biogeochemical recycling of carbon and associated elements along tropical coastal region.

Since Despites the decomposition rates which vary considerably among different mangrove species and the detritus build up based on diverse environmental conditions, the range of average tides of the area, species richness of litter fall may be important in the determination of the nature of relationships between biodiversity and ecosystem properties [12]. Mangrove forests with more frequent tides and river inundations are assumed to be more productive [13]. This assumption has been widely explored with studies carried out in diverse geomorphologic settings to ascertain the linkage between productivity and specific types of geophysical processes of coastal environmental settings. Current knowledge on the productivity of mangrove ecosystems based on studies from North America, Australia and South East Asia, has revealed variability in the productivity of mangrove ecosystems. Various opinions have been advanced for the main causes of the variation such as methodology employed and uncertainties [14]. These opinions require precise measurements of local systems including production, for more accurate predictions [15]. Some data gaps exist in knowledge on the relative importance of litterfall to the overall productivity of mangrove and the adjacent marine ecosystems. This demands a better understanding of the dynamics of the diverse local systems by careful sampling of all necessary components of net primary productivity of mangrove ecosystems. Since mangroves play a major role in the dissolved organic matter exchange between land and ocean, their rapid decline in recent decades could reduce the flux of terrestrial dissolved organic matter to ocean, impacting one of the largest organic carbon pools on earth with potential consequences for atmospheric composition and climate [16]. The quantification of leaf litterfall, decomposition and biochemical contents of mangrove species in the riverine mangrove forests, of the Cross River estuary is important for estimating the productivity of the system and its relevance for food webs in the Cross River estuary and thus important in developing management strategies for sustainable use of the mangroves. The biological productivity of the Cross River estuarine water and its controlling factors has been studied [17, 18]. However, information on the productivity of the fringing mangrove forests of the estuary, the trophic interactions and material flow within the ecosystem remain largely unstudied. Thus, the significance of the mangrove forests in the overall biological productivity of this estuary remains vague. A study of litter decomposition will give insight into the rates of productivity and mineralization in the mangrove ecosystem. The objectives of this study include investigating the temporal and spatial variation of decomposition and nutrient contents of mangrove leaves as well as to identify differences among mangrove species in response to biotic and abiotic factors which vary across intertidal zones and to compare the results obtained with those of other tropical mangrove ecosystems in other regions of the world.

\section{Materials and Methods}

\subsection{Geo-Morphological Description of the Study Area}

The study area covered is the mangal forest vegetation of the Great Kwa River, in Esuk Mba east of the Cross River estuary which flows into the Gulf of Guinea (FIG. 1). This area lies within latitudes $04^{\circ} 45^{\prime}$ and $04^{\circ} 15^{\prime}$ North of the Equator and longitudes $008^{\circ} 15^{\prime}$ and $008^{\circ} 30^{\prime}$ East of Greenwich Meridian along the eastern border of the University of Calabar. Geomorphologically, the area is characterized by silty clays, peaty clays commonly called "Chikoko soil", saline sands and mud flat benthic sediment. These edaphic structures are intermittently inundated by the ebbing and up-rising tidal flow of hydrological regimes and also characterized by a sulphate odour, which becomes more intensive at a high temperature and ebbing tide. More conspicuous with mycoflora and cyanophyta (blue-green algae) at the ebbing tide. The mangal forest vegetation in this region occurs in clear zonation pattern along a tidal gradient with $N$. fruticans forming the outermost zone towards the water front, followed by either pure stands of $R$ racemosa or mixed stands of $R$. racemosa and $N$. fruticans. These zones are followed by pure stands of $A$. africana. Climate in this region is equatorial and is characterized by a pattern of alternating wet and dry seasons. The wet 
season extends from April to September and the dry season from October to March with maximum temperature of $34.1^{\mathrm{O}} \mathrm{C}$ to a minimum of $22.2^{\mathrm{O}} \mathrm{C}$ and relative humidity in the range of $70-92 \%$ [19].

\subsection{Species Assessment, Leaf Decomposition, Total Carbon and Nitrogen Determination}

Three sites were established along a tidal gradient within the mixed mangrove forest. The study sites chosen represent the zonation along the gradient levels of low tide (LTL), mid tide level (MTL) and the high tide level (HTL) dominated by $N$. fruticans, $R$. racemosa and A. africana respectively.

Leaf decomposition rates of $N$. fruticans, $R$. racemosa and $A$. africana were determined as loss of dry mass from litter bags over a period of eight weeks at the three tidal levels (LTL, MTL and HTL). Litter bags were colour coded with nylon ropes to facilitate field differentiation of species/samples. Senescent leaves were collected by shaking the trees and picked off the trees. The senescent leaves samples were rinsed with deionized water and airdried for $24 \mathrm{~h}$ to remove salt and surface water. The airdried leaves were weighed $(20 \mathrm{~g})$ and placed in nylon litter bags $(30 \times 30 \mathrm{~cm})$ of $1 \mathrm{~mm}$ mesh [20]. This mesh size design allows for microbial decomposition with the exclusion of large consumers such as crabs, and prevention of loss of particulate organic matter during wash-outs.

To investigate the effects of species, tidal level and exposure time, on decomposition rate, fifteen (15) bags of each species ( $N$. fruticans, $R$. racemosa and $A$. africana) were placed at each of the three tidal levels (LTL, MTL, and HTL) for eight (8) weeks. The bags were securely tied to aerial roots such that they laid flat on the sediments. To compare decomposition rates of species over different exposure time intervals, three bags of each species were randomly retrieved at intervals fortnightly $(14,28,42$ and 56 days) from each tidal level. During each sampling, 27 litter bags - species (3) x tidal level (3) x replicates (3) were retrieved. A total of 108 bags were sampled. All samples were returned to the laboratory where they were rinsed with deionized water, oven dried to constant mass at $80^{\circ} \mathrm{C}$.

To derive the wet: dry weight ratio for the leaves placed in the field, $20 \mathrm{~g}$ of samples of senescent leaves for the range of leaf sizes used for the experiments were retained. The leaves were rinsed with deionized water, air-dried for $24 \mathrm{~h}$ and weighed. The air-dried leaves were oven-dried to constant mass. These were then used to determine the initial dry mass of leaves in the litter bags for each species. The dried leaves were ground for each species and used to determine the total carbon content using the Walkey-Black wet oxidation-method and total nitrogen content using the Kjeldahl method [21].

\subsection{Data Analysis}

\subsubsection{Leaf Decomposition Rates}

Graphs of mean dry mass remaining in litter bags after time as a percentage of initial dry mass were obtained for all species at each tidal level. Triplicate decay coefficients were used to test for the effects of species and tidal level on leaf decomposition rates. A negative single exponential decay model by Minderman [22] was used to test for relative differences in decomposing rates among species, exposure times and tidal gradients and to express the relationship between percentage dry mass remaining in litter bags and sampling time for all species at the three tidal levels using the equation.

$$
\frac{\omega_{t}}{\omega_{o}}=e^{-K_{d} t}
$$

where $\omega_{o}=$ initial dry mass

$\omega_{1}=$ mass remaining at time $\mathrm{t}$

$\mathrm{K}_{\mathrm{d}}=$ decomposition coefficient in days $\left(\mathrm{d}^{-1}\right)$.

The parameter $K_{d}$ was estimated for each species. The effects of species, tidal level and exposure time on the rate of decomposition were assessed using three-way analyses of variance (ANOVA) by Obi [23], the main effects being species, tidal level and time. The times required for the decomposition of half the initial material $\left(t_{50}\right)$ was determined using the equation.

$$
t_{50}=\operatorname{In} \frac{2}{K}
$$

where $I n=$ natural logarithm

$\mathrm{K}=$ decomposition rate

\subsubsection{Net Changes In Nutrients}

The nutrient accumulation index (NAI) for each species at the tidal levels were calculated using the equation by Harmon et al., [24] in order to characterize the overall pattern of net mineralization or accumulation of carbon and nitrogen in the decomposing leaves.

$$
N A I=\frac{\omega_{t} x_{t}}{\omega_{o} x_{o}}
$$

where $\omega_{\mathrm{t}}=$ the dry weight of the leaf litter at time $\mathrm{t}$,

$\mathrm{x}_{\mathrm{t}}=$ the initial nutrient concentration of the leaf litter at time $\mathrm{t}$,

$\omega_{0}=$ the initial dry weight of the leaf litter and

$\mathrm{x}_{\mathrm{o}}=$ the initial concentration of nutrient in the leaf litter.

An NAI value of 1.0 indicates that the decomposed leaf litter contains the same mass of the element ' $\mathrm{X}$ ' when the leaf litter was placed in the litter bag; NAI $<1.0$ indicates net mineralization of the element from the decaying leaf litter and NAI $>1.0$ indicates net assimilation of the element by the decaying leaf litter.

Data obtained from total carbon and total nitrogen determination were used in calculating $\mathrm{C}: \mathrm{N}$ ratio for each species at the tidal levels for the decomposing leaf litter. 


\section{Results}

\subsection{Leaf Decomposition}

The results of the average percentage of original leaf dry weight remaining following exposure at specific time intervals for three dominant mangal species $(N$. fruticans, $R$. racemosa and A. africana) across tidal gradients (low, mid, high) are presented (Table 1). The results show that decomposition rates varied among the species and across tidal levels. Results of the three way analyses of variance (ANOVA) showed that there were highly significant differences $(\mathrm{P}<0.001)$ in decomposition rate among the species, exposure time and tidal levels (Table 2). However, there were neither significant interaction effects between the species and exposure times and tidal levels, nor was there 3-way interaction amongst them suggesting that all the species were affected in the same way (Table 2).

Table 1. Leaf decomposition of mangrove species (dry weight mean \pm standard error) in litter bags following exposure for 14, 28, 42 and 56 days at Esuk Mba of Great Kwa River, Cross River estuary, Nigeria litter bags for each (Avicennia africana, Rhizophora racemosa Nypa fruticans,) were placed in a field across tidal gradients.

\begin{tabular}{|c|c|c|c|c|c|c|c|c|c|c|}
\hline Species & $\begin{array}{l}\text { Exposure } \\
\text { (days) }\end{array}$ & $\begin{array}{l}\text { Tidal } \\
\text { level }\end{array}$ & $\begin{array}{l}\text { Dry weight } \\
\text { remaining (g) }\end{array}$ & $\begin{array}{c}\% \text { Dry } \\
\text { weight } \\
\text { remaining }\end{array}$ & $\begin{array}{c}\text { Decay } \\
\text { coefficient } \\
\left(K^{\mathrm{d}-1}\right)\end{array}$ & $\begin{array}{c}\% \\
\text { Nitrogen } \\
\text { content }\end{array}$ & $\begin{array}{c}\text { NAI - } \\
\text { Nitrogen }\end{array}$ & $\begin{array}{c}\% \text { Carbon } \\
\text { content }\end{array}$ & $\begin{array}{c}\text { NAI- } \\
\text { Carbon }\end{array}$ & $\mathbf{C} / \mathbf{N}$ \\
\hline \multirow[t]{19}{*}{ A. fruticans } & \multirow[t]{3}{*}{0} & Low & $4.37 \pm 0$ & $100.00 \pm 0$ & $0 \pm 0$ & $1.08 \pm 0$ & $1.00 \pm 0$ & $29.92 \pm 0$ & $1.00 \pm 0$ & 27.7 \\
\hline & & Mid & $4.37 \pm 0$ & $100.00 \pm 0$ & $0 \pm 0$ & $1.08 \pm 0$ & $1.00 \pm 0$ & $29.92 \pm 0$ & $1.00 \pm 0$ & 27.7 \\
\hline & & High & $4.37 \pm 0$ & $100.00 \pm 0$ & $0 \pm 0$ & $1.08 \pm 0$ & $1.00 \pm 0$ & $29.92 \pm 0$ & $1.00 \pm 0$ & 27.7 \\
\hline & \multirow[t]{3}{*}{14} & Low & $3.29 \pm 0.18$ & $75.37 \pm 4.22$ & $0.0205 \pm 0.004$ & $1.07 \pm 0.05$ & $0.75 \pm 0.08$ & $30.84 \pm 2.07$ & $0.782 \pm 0.09$ & 28.8 \\
\hline & & Mid & $3.28 \pm 0.00$ & $75.06 \pm 1.85$ & $0.0206 \pm 0.001$ & $1.14 \pm 0.08$ & $30.84 \pm 2.07$ & $0.75 \pm 0.08$ & $0.782 \pm 0.09$ & 24.7 \\
\hline & & High & $3.84 \pm 0.17$ & $87.95 \pm 3.82$ & $0.0093 \pm 0.003$ & $1.03 \pm 0.06$ & $0.84 \pm 0.01$ & $31.17 \pm 0.07$ & $0.92 \pm 0.04$ & 30.3 \\
\hline & \multirow[t]{3}{*}{28} & Low & $3.05 \pm 0.14$ & $69.72 \pm 3.09$ & $0.012 \pm 0.0016$ & $1.15 \pm 0.03$ & $0.74 \pm 0.05$ & $31.18 \pm 0.13$ & $0.73 \pm 0.03$ & 27.1 \\
\hline & & Mid & $3.33 \pm 0.02$ & $76.20 \pm 0.39$ & $0.0097 \pm 0.0002$ & $1.18 \pm 0.03$ & $0.83 \pm 0.02$ & $31.98 \pm 0.41$ & $0.81 \pm 0.01$ & 27.1 \\
\hline & & High & $3.53 \pm 0.17$ & $80.78 \pm 3.82$ & $0.0077 \pm 0.0017$ & $1.14 \pm 0.02$ & $0.85 \pm 0.05$ & $32.32 \pm 0.12$ & $0.87 \pm 0.04$ & 28.1 \\
\hline & \multirow[t]{3}{*}{42} & Low & $2.83 \pm 0.21$ & $64.83 \pm 4.85$ & $0.015 \pm 0.0018$ & $1.27 \pm 0.01$ & $0.77 \pm 0.06$ & $23.44 \pm 1.24$ & $0.51 \pm 0.06$ & 18.5 \\
\hline & & Mid & $3.08 \pm 0.26$ & $70.48 \pm 5.96$ & $0.0085 \pm 0.002$ & $1.28 \pm 0.02$ & $0.83 \pm 0.06$ & $22.41 \pm 0.69$ & $0.53 \pm 0.03$ & 17.5 \\
\hline & & High & $3.20 \pm 0.19$ & $79.23 \pm 4.38$ & $0.0075 \pm 0.001$ & $1.24 \pm 0.02$ & $0.84 \pm 0.04$ & $23.04 \pm 0.58$ & $0.56 \pm 0.04$ & 18.6 \\
\hline & \multirow[t]{3}{*}{56} & Low & $2.38 \pm 0.05$ & $54.46 \pm 1.21$ & $0.0109 \pm 0.003$ & $1.45 \pm 0.01$ & $0.53 \pm 0.09$ & $24.25 \pm 0.37$ & $0.32 \pm 0.06$ & 16.7 \\
\hline & & Mid & $2.38 \pm 0.05$ & $64.46 \pm 1.21$ & $0.0109 \pm 0.003$ & $1.42 \pm 0.01$ & $0.72 \pm 0.01$ & $23.92 \pm 0.52$ & $0.44 \pm 0.01$ & 16.9 \\
\hline & & High & $2.56 \pm 0.12$ & $58.51 \pm 2.62$ & $0.0096 \pm 0.000$ & $1.43 \pm 0.01$ & $0.77 \pm 0.03$ & $24.09 \pm 0.49$ & $0.47 \pm 0.01$ & 16.9 \\
\hline & \multirow[t]{3}{*}{ Mean } & Low & $2.73 \pm 0.20$ & $62.39 \pm 4.46$ & $0.0153 \pm 0.0017$ & $1.24 \pm 0.05$ & $0.69 \pm 0.04$ & $27.43 \pm 1.20$ & $0.59 \pm 0.06$ & 22.1 \\
\hline & & Mid & $69.05 \pm 2.95$ & $69.05 \pm 2.95$ & $0.0124 \pm 0.0016$ & $1.26 \pm 0.04$ & $0.97 \pm 0.03$ & $26.61 \pm 1.15$ & $0.62 \pm 0.05$ & 21.1 \\
\hline & & High & $3.29 \pm 0.16$ & $75.12 \pm 3.65$ & $0.0085 \pm 0.0000$ & $1.21 \pm 0.05$ & $0.83 \pm 0.02$ & $27.66 \pm 1.26$ & $0.71 \pm 0.06$ & 22.9 \\
\hline & \multicolumn{2}{|c|}{ Overall mean } & $3.01 \pm 0.10$ & $68.85 \pm 2.31$ & $0.0121 \pm 0.0000$ & $1.23 \pm 0.03$ & $0.77 \pm 0.02$ & $27.23 \pm 0.68$ & $0.64 \pm 0.03$ & 22.1 \\
\hline \multirow[t]{19}{*}{ R. racemosa } & \multirow[t]{3}{*}{0} & Low & $6.54 \pm 0$ & $100.00 \pm 0$ & $0 \pm 0$ & $0.80 \pm 0$ & $1.00 \pm 0$ & $29.15 \pm 0$ & $1.00 \pm 0$ & 36.4 \\
\hline & & Mid & $6.54 \pm 0$ & $100.00 \pm 0$ & $0 \pm 0$ & $0.80 \pm 0$ & $1.00 \pm 0$ & $29.15 \pm 0$ & $1.00 \pm 0$ & 36.4 \\
\hline & & High & $6.54 \pm 0$ & $100.00 \pm 0$ & $0 \pm 0$ & $0.80 \pm 0$ & $1.00 \pm 0$ & $29.15 \pm 0$ & $1.00 \pm 0$ & 36.4 \\
\hline & \multirow[t]{3}{*}{14} & Low & $5.48 \pm 0.02$ & $84.26 \pm 0.72$ & $0.012 \pm 0.0008$ & $0.95 \pm 0.01$ & $0.99 \pm 0.01$ & $25.46 \pm 0.07$ & $0.73 \pm 0.01$ & 26.8 \\
\hline & & Mid & $5.75 \pm 0.26$ & $87.73 \pm 4.01$ & $0.0093 \pm 0.0031$ & $0.87 \pm 0.05$ & $0.95 \pm 0.04$ & $27.06 \pm 0.24$ & $0.82 \pm 0.03$ & 31.1 \\
\hline & & High & $5.84 \pm 0.14$ & $89.51 \pm 2.25$ & $0.0082 \pm 0.0017$ & $0.93 \pm 0.02$ & $1.04 \pm 0.03$ & $26.39 \pm 0.23$ & $0.81 \pm 0.03$ & 28.4 \\
\hline & \multirow[t]{3}{*}{28} & Low & $6.15 \pm 0.21$ & $78.79 \pm 3.17$ & $0.0086 \pm 0.0015$ & $0.99 \pm 0.05$ & $0.98 \pm 0.04$ & $23.34 \pm 1.27$ & $0.65 \pm 0.05$ & 23.6 \\
\hline & & Mid & $5.34 \pm 0.09$ & $81.60 \pm 1.43$ & $0.073 \pm 0.0007$ & $1.02 \pm 0.02$ & $1.04 \pm 0.02$ & $28.87 \pm 0.18$ & $0.67 \pm 0.01$ & 28.3 \\
\hline & & High & $5.74 \pm 0.24$ & $87.72 \pm 3.65$ & $0.048 \pm 0.0015$ & $0.99 \pm 0.03$ & $1.09 \pm 0.02$ & $24.14 \pm 0.49$ & $0.73 \pm 0.19$ & 24.4 \\
\hline & \multirow[t]{3}{*}{42} & Low & $4.90 \pm 0.13$ & $65.42 \pm 2.27$ & $0.0103 \pm 0.0007$ & $1.04 \pm 0.01$ & $0.85 \pm 0.02$ & $22.39 \pm 0.23$ & $0.49 \pm 0.02$ & 21.5 \\
\hline & & Mid & $4.90 \pm 0.07$ & $74.92 \pm 1.07$ & $0.0066 \pm 0.0007$ & $1.03 \pm 0.01$ & $0.97 \pm 0.01$ & $23.28 \pm 0.54$ & $0.59 \pm 0.02$ & 24.0 \\
\hline & & High & $6.28 \pm 0.03$ & $80.60 \pm 0.52$ & $0.0051 \pm 0.0002$ & $0.98 \pm 0.00$ & $0.99 \pm 0.00$ & $25.00 \pm 0.17$ & $0.69 \pm 0.01$ & 25.3 \\
\hline & \multirow[t]{3}{*}{56} & Low & $3.92 \pm 0.03$ & $59.99 \pm 0.36$ & $0.0091 \pm 0.0001$ & $1.03 \pm 0.01$ & $0.77 \pm 0.01$ & $20.88 \pm 0.06$ & $0.43 \pm 0.00$ & 27.1 \\
\hline & & Mid & $4.14 \pm 0.08$ & $63.26 \pm 1.23$ & $0.0082 \pm 0.0004$ & $1.02 \pm 0.01$ & $0.81 \pm 0.01$ & $21.15 \pm 0.12$ & $0.46 \pm 0.01$ & 26.1 \\
\hline & & High & $4.36 \pm 0.03$ & $66.72 \pm 0.45$ & $0.0072 \pm 0.0001$ & $1.01 \pm 0.00$ & $0.85 \pm 0.01$ & $21.29 \pm 0.26$ & $0.49 \pm 0.01$ & 25.1 \\
\hline & \multirow[t]{3}{*}{ Mean } & Low & $4.70 \pm 0.19$ & $72.12 \pm 3.07$ & $0.0102 \pm 0.0006$ & $1.01 \pm 0.02$ & $0.89 \pm 0.03$ & $23.02 \pm 0.57$ & $0.57 \pm 0.04$ & 22.8 \\
\hline & & Mid & $5.03 \pm 0.19$ & $76.88 \pm 2.90$ & $0.0078 \pm 0.0008$ & $0.99 \pm 0.02$ & $0.94 \pm 0.03$ & $23.84 \pm 0.65$ & $0.64 \pm 0.04$ & 25.4 \\
\hline & & High & $5.30 \pm 0.19$ & $81.14 \pm 2.86$ & $0.0063 \pm 0.0007$ & $0.98 \pm 0.01$ & $0.99 \pm 0.03$ & $24.20 \pm 0.58$ & $0.68 \pm 0.04$ & 24.7 \\
\hline & \multicolumn{2}{|c|}{ Overall mean } & $5.01 \pm 0.12$ & $76.71 \pm 1.77$ & $0.0081 \pm 0.0005$ & $0.99 \pm 0.01$ & $0.94 \pm 0.02$ & $23.69 \pm 0.35$ & $0.63 \pm 0.02$ & 23.9 \\
\hline N. fruticans & 0 & Low & $12.49 \pm 0$ & $100.00 \pm 0$ & $0 \pm 0$ & $0.87 \pm 0$ & $1.00 \pm 0$ & $28.53 \pm 0$ & $1.00 \pm 0$ & 32.8 \\
\hline
\end{tabular}




\begin{tabular}{|c|c|c|c|c|c|c|c|c|c|c|}
\hline Species & $\begin{array}{c}\text { Exposure } \\
\text { (days) }\end{array}$ & $\begin{array}{l}\text { Tidal } \\
\text { level }\end{array}$ & $\begin{array}{l}\text { Dry weight } \\
\text { remaining (g) }\end{array}$ & $\begin{array}{c}\% \text { Dry } \\
\text { weight } \\
\text { remaining }\end{array}$ & $\begin{array}{c}\text { Decay } \\
\text { coefficient } \\
\left(\mathbf{K}^{\mathrm{d}-1}\right)\end{array}$ & $\begin{array}{c}\% \\
\text { Nitrogen } \\
\text { content }\end{array}$ & $\begin{array}{c}\text { NAI - } \\
\text { Nitrogen }\end{array}$ & $\begin{array}{c}\text { \% Carbon } \\
\text { content }\end{array}$ & $\begin{array}{l}\text { NAI- } \\
\text { Carbon }\end{array}$ & $\mathbf{C} / \mathbf{N}$ \\
\hline & \multirow{5}{*}{14} & Mid & $12.49 \pm 0$ & $100.00 \pm 0$ & $0 \pm 0$ & $0.87 \pm 0$ & $1.00 \pm 0$ & $28.53 \pm 0$ & $1.00 \pm 0$ & 32.8 \\
\hline & & High & $12.49 \pm 0$ & $100.00 \pm 0$ & $0 \pm 0$ & $0.87 \pm 0$ & $1.00 \pm 0$ & $28.53 \pm 0$ & $1.00 \pm 0$ & 32.8 \\
\hline & & Low & $10.62 \pm 0.31$ & $85.05 \pm 2.48$ & $0.0116 \pm 0.0020$ & $0.87 \pm 0.02$ & $0.85 \pm 0.02$ & $26.65 \pm 0.13$ & $0.78 \pm 0.03$ & 31.4 \\
\hline & & Mid & $10.80 \pm 0.05$ & $86.47 \pm 0.39$ & $0.0104 \pm 0.0003$ & $0.83 \pm 0.01$ & $0.83 \pm 0.01$ & $27.98 \pm 0.14$ & $0.85 \pm 0.01$ & 33.7 \\
\hline & & High & $10.83 \pm 0.10$ & $86.74 \pm 0.83$ & $0.0102 \pm 0.0007$ & $0.86 \pm 0.03$ & $0.82 \pm 0.01$ & $28.25 \pm 0.13$ & $0.86 \pm 0.01$ & 32.9 \\
\hline & \multirow[t]{3}{*}{28} & Low & $10.48 \pm 0.09$ & $83.88 \pm 0.79$ & $0.0062 \pm 0.0004$ & $1.03 \pm 0.00$ & $0.98 \pm 0.01$ & $22.41 \pm 0.52$ & $0.66 \pm 0.01$ & 21.8 \\
\hline & & Mid & $10.46 \pm 0.12$ & $83.72 \pm 0.95$ & $0.0063 \pm 0.0004$ & $1.03 \pm 0.01$ & $0.99 \pm 0.02$ & $25.46 \pm 0.057$ & $0.75 \pm 0.01$ & 24.7 \\
\hline & & High & $10.67 \pm 0.06$ & $85.40 \pm 0.52$ & $0.0057 \pm 0.0002$ & $0.97 \pm 0.01$ & $0.95 \pm 0.01$ & $256.02 \pm 0.13$ & $0.75 \pm 0.01$ & 25.8 \\
\hline & \multirow[t]{3}{*}{42} & Low & $9.43 \pm 0.07$ & $75.50 \pm 0.58$ & $0.0067 \pm 0.0002$ & $1.03 \pm 0.01$ & $0.89 \pm 0.00$ & $19.59 \pm 0.32$ & $0.52 \pm 0.01$ & 19.0 \\
\hline & & Mid & $9.94 \pm 0.12$ & $79.58 \pm 0.97$ & $0.0054 \pm 0.0003$ & $1.00 \pm 0.02$ & $0.92 \pm 0.02$ & $20.01 \pm 0.06$ & $0.55 \pm 0.01$ & 20.0 \\
\hline & & High & $10.05 \pm 0.12$ & $80.45 \pm 0.96$ & $0.0052 \pm 0.0003$ & $0.96 \pm 0.02$ & $0.89 \pm 0.01$ & $19.95 \pm 0.23$ & $0.56 \pm 0.01$ & 20.8 \\
\hline & \multirow[t]{3}{*}{56} & Low & $9.01 \pm 0.21$ & $72.17 \pm 0.69$ & $0.0058 \pm 0.0004$ & $1.03 \pm 0.01$ & $0.86 \pm 0.03$ & $18.62 \pm 0.28$ & $0.47 \pm 0.02$ & 18.7 \\
\hline & & Mid & $9.26 \pm 0.04$ & $74.11 \pm 0.35$ & $0.0053 \pm 0.0001$ & $1.02 \pm 0.02$ & $0.86 \pm 0.02$ & $20.15 \pm 0.20$ & $0.52 \pm 0.01$ & 19.8 \\
\hline & & High & $9.68 \pm 0.08$ & $77.50 \pm 0.62$ & $0.0046 \pm 0.0001$ & $1.02 \pm 0.04$ & $0.88 \pm 0.00$ & $20.21 \pm 0.23$ & $0.55 \pm 0.01$ & 19.8 \\
\hline & \multirow[t]{3}{*}{ Mean } & Low & $9.89 \pm 0.22$ & $79.15 \pm 1.78$ & $0.0076 \pm 0.0009$ & $0.99 \pm 0.02$ & $0.89 \pm 0.02$ & $21.82 \pm 0.95$ & $0.61 \pm 0.03$ & 22.0 \\
\hline & & Mid & $10.11 \pm 0.18$ & $80.97 \pm 1.44$ & $0.0069 \pm 0.0006$ & $0.97 \pm 0.02$ & $0.89 \pm 0.02$ & $23.39 \pm 1.05$ & $0.67 \pm 0.04$ & 24.1 \\
\hline & & High & $10.31 \pm 0.15$ & $82.52 \pm 1.17$ & $0.0064 \pm 0.0007$ & $0.95 \pm 0.02$ & $0.89 \pm 0.02$ & $23.36 \pm 1.05$ & $0.68 \pm 0.04$ & 24.6 \\
\hline & \multicolumn{2}{|c|}{ Overall mean } & $10.10 \pm 0.11$ & $80.88 \pm 0.86$ & $0.0069 \pm 0.0004$ & $0.97 \pm 0.01$ & $0.89 \pm 0.01$ & $22.86 \pm 0.58$ & $0.65 \pm 0.02$ & 23.6 \\
\hline
\end{tabular}

$\mathrm{NAI}=$ Nutrient accumulation index, $\mathrm{C} / \mathrm{N}=$ Carbon Nitrogen ratio

Table 2. Three-way analysis of variance (ANOVA) decomposition of mangal leaves, (N. fruticans, $R$. racemosa and A. africana) in litter bags placed at three tidal levels (low, mid, high) at Esuk Mba of the Great Kwa River of Cross River estuary, Nigeria.

\begin{tabular}{|c|c|c|c|c|c|c|c|c|c|c|c|c|c|}
\hline \multicolumn{5}{|c|}{ Dependent variable: (a) Dry wt remaining } & \multicolumn{3}{|c|}{ (b) $\%$ Nitrogen Content } & \multicolumn{3}{|c|}{ (c) $\%$ Carbon Content } & \multicolumn{3}{|c|}{$\begin{array}{l}\text { (d) Decay Coefficient } \\
\text { (Log Transformed) }\end{array}$} \\
\hline $\begin{array}{l}\text { Source of } \\
\text { variation }\end{array}$ & $\mathrm{df}$ & MS & $\mathrm{F}$ & $\mathrm{P}$ & MS & $\mathrm{F}$ & $\mathrm{P}$ & MS & $\mathrm{F}$ & $\mathrm{P}$ & MS & $\mathrm{F}$ & $\mathrm{P}$ \\
\hline Species & 2 & 481.37 & 7000.709 & $0 * * *$ & 0.776 & 342.188 & $0 * * *$ & 194.318 & 208.696 & $0 * * *$ & 2.498 & 34.48 & $0 * * *$ \\
\hline $\begin{array}{l}\text { Exposure } \\
\text { (days) }\end{array}$ & 3 & 10.64 & 154.745 & $0 * * *$ & 0.205 & 90.521 & $0 * * *$ & 276.227 & 296.666 & $0 * * *$ & 1.35 & 18.631 & $0 * * *$ \\
\hline Tidal level & 2 & 2.496 & 36.293 & $0 * * *$ & 0.009 & 3.927 & $0.024 *$ & 8.784 & 9.434 & $0 * * *$ & 1.61 & 22.223 & $0 * * *$ \\
\hline \multicolumn{14}{|l|}{ Species X } \\
\hline Exposure days & 6 & 0.147 & 2.136 & $0.059 \mathrm{~ns}$ & 0.041 & 18.287 & $0 * * *$ & 34.099 & 36.622 & $0 * * *$ & 0.193 & 2.662 & $0.022 *$ \\
\hline $\begin{array}{l}\text { Species x } \\
\text { Tidal level }\end{array}$ & 4 & 0.027 & 0.389 & $0.816 \mathrm{~ns}$ & 0.002 & 0.855 & $0.495 \mathrm{~ns}$ & 4.527 & 4.862 & $0.002 * *$ & 0.152 & 2.097 & $0.09 \mathrm{~ns}$ \\
\hline \multicolumn{14}{|l|}{ Exposure days $\mathrm{x}$} \\
\hline $\begin{array}{l}\text { Tidal level } \\
\text { Species } \mathrm{x}\end{array}$ & 6 & 0.075 & 1.094 & $0.374 \mathrm{~ns}$ & 0.002 & 0.725 & $0.631 \mathrm{~ns}$ & 1.123 & 1.206 & $0.313 \mathrm{~ns}$ & 0.027 & 0.376 & $0.892 \mathrm{~ns}$ \\
\hline \multicolumn{14}{|l|}{ Exposure days $\mathrm{x}$} \\
\hline Tidal level & 12 & 0.075 & 1.084 & $0.386 \mathrm{~ns}$ & 0.002 & 0.969 & $0.486 \mathrm{~ns}$ & 1.644 & 1.766 & $0.071 \mathrm{~ns}$ & 0.081 & 1.118 & $0.359 \mathrm{~ns}$ \\
\hline Error & 72 & 0.069 & & & 0.002 & & & 0.931 & & & 0.072 & & \\
\hline
\end{tabular}

$* * *=$ Significant difference $(\mathrm{P}<0.001) \mathrm{df}=$ Degrees of freedom

$*=$ Significant differences $(\mathrm{P} \leq 0.05) \mathrm{F}=$ Variance ratio
$* *=$ Significant difference $(\mathrm{P} \leq 0.05 \mathrm{MS}=$ Mean squares $\mathrm{ns}=$ not significant $\mathrm{P}=$ Level of probability
The ANOVA showed that the decomposition rates were species specific. There was a highly significant effect of species on decomposition rates of mangrove leaf litter $(\mathrm{P}<$ $0.001)$. $R$. racemosa demonstrated the lowest rate of decomposition at $0.012 \pm 0.001\left(\mathrm{~g} \mathrm{~d}^{-1}\right)$ compared to $N$. fruticans at $0.008 \pm 0.001\left(\mathrm{~g} \mathrm{~d}^{-1}\right)$ and A. africana at $0.007 \pm$ $0.004\left(\mathrm{~g} \mathrm{~d}^{-1}\right)$ (Table 1). There was a significant interaction effect for species and exposure time $(\mathrm{P} \leq 0.05)$. There was no significant interaction between species and tidal levels. However decomposition rates generally increased from high tide to low tide levels (Table 1). Relative differences of the decomposition rates among the species within the forest are readily apparent, being highest at the low tide level compared to other tidal levels (Table 2).

\subsection{Leaf Litter Turnover}

The estimated turnover rate $\left(\mathrm{K}_{\mathrm{t}}\right)$ based on the relative measure of leaf fall and leaf litter on the forest floor was compared to the coefficients of projected leaf litter turnover $\left(\mathrm{K}_{\mathrm{d}}\right)$ based on rates of leaf decomposition (Table 1), each 
coefficient representing a measure of the leaf litter biomass that is lost per day. The actual residence times of leaf litter based on turnover rate (Table 3) at the three tidal levels were much shorter than the projected times based on the leaf decomposition rates (Table 4). The projected residence time and times required for the decomposition of half the initial leaf litter $\left(\mathrm{T}_{50}\right)$ among the species across the tidal gradients, based on $\mathrm{Kd}$ ranged from 83 to 142 days while the actual residence times based on $\mathrm{Kt}$ were less than one day (Table 3) across the tidal gradients.. Leaves of $A$. africana had the shortest projected residence time with half life of $46 \mathrm{~d}$ at low tide, $57 \mathrm{~d}$ at mid tide and $77 \mathrm{~d}$ at high tide compared to $R$. racemosa with half life of $69 \mathrm{~d}$ at low tide, $86 \mathrm{~d}$ at mid tide and $115 \mathrm{~d}$ at high tide, and $N$. fruticans with half life of $86 \mathrm{~d}$ at low tide, $99 \mathrm{~d}$ at mid tide and $115 \mathrm{~d}$ at high tide (Table 4). Thus, the $\mathrm{K}_{\mathrm{t}}$ turnover rates are much higher than the $\mathrm{K}_{\mathrm{d}}$ turnover rates and the relative differences in leaf litter turnover are also different between the two methods across tidal gradients. The residence time of leaf litter (actual or projected) in general was shortest at the low tide level (Table 5).

\subsection{Nutrient Dynamics}

The percentage nitrogen and carbon contents in decomposing leaf litter were determined. The nitrogen content in decomposing leaves increased while carbon content decreased with time for all species at all the tidal levels during the 56 day study period. Analyses of variance (ANOVA) showed highly significant differences $(\mathrm{P}<0.001)$ in the content of nitrogen and carbon (Table 2) among the leaves. Leaves of $A$. africana had the highest initial nitrogen content of $1.08 \%$ (dry weight) and a $\mathrm{C}: \mathrm{N}$ ratio of
27.7 compared to $N$. fruticans with $0.87 \%$ nitrogen (dry weight), C:N of 32.8 and $R$. racemosa with $0.80 \%$ nitrogen (dry weight) and $\mathrm{C}: \mathrm{N}$ of 36.4 (Table 1). There was also a significant effect of time (exposure days) on the nitrogen and carbon contents of the decomposing leaf litter $(\mathrm{P}=$ 0.001). The nitrogen content increased with longer exposure while the carbon content decreased with longer exposure. The tidal level also had significant effect on the nitrogen content $(\mathrm{P} \leq 0.05)$ and carbon content $(\mathrm{P}<0.001)$ of the decomposing leaf litter. There was a significant interaction between species and time $(\mathrm{P}<0.001)$ (Table 2$)$.

Linear regression analyses of nutrient contents of decomposing litter on time indicated that nitrogen content significantly increased, while carbon content significantly decreased with time, the slopes significantly different from 0 at $\mathrm{P}<0.001$. The results obtained indicate that the duration of the decomposition affects the nutrient contents. At the end of the decomposition study ( 2 months), mean carbon C:N ratio decreased from 27.7 to $22.1,36.4$ to 23.9 and 32.8 to 23.6 in A. africana, $R$. racemosa and $N$. fruticans respectively (Table 1$)$.

Calculations of net changes in nutrients (immobilization vs mineralization) in the decomposing litter revealed differences among species and across tidal levels. There was a net mineralization of nutrients (nitrogen and carbon) in A. africana, and N. fruticans at the three tidal levels, and net immobilization of nitrogen for $R$. racemosa at the high tide level. Overall species across tidal gradients showed a net mineralization of nitrogen and carbon in all the decomposing leaf litter (Table 1).

Table 3. Estimate of mean litter turnover across tidal gradients in (low, mid and high) in a mixed mangrove forest (N. fruticans, R. racemosa and A. africana) at Esuk Mba of the Great Kwa River of Cross River estuary, Nigeria.

\begin{tabular}{|c|c|c|c|c|c|c|}
\hline $\begin{array}{l}\text { Tidal } \\
\text { level }\end{array}$ & Component & $\begin{array}{c}\text { Litterfall (LF) / } \\
\text { g m}^{-2}(\mathbf{d w t})\end{array}$ & $\begin{array}{l}\text { Litter biomass } \\
\text { (LB) }\left(\mathrm{g} \mathrm{m}^{-2} \mathrm{dwt}\right)\end{array}$ & $\begin{array}{c}\text { Turnover rate }\left(K_{t}\right) \\
(L F / L B)\end{array}$ & $\begin{array}{l}\text { Residence time } \\
\left(1 / K_{t}\right) \text { (days) }\end{array}$ & $\begin{array}{c}\text { Half-life } \\
\left(\mathbf{T}_{50}\right) \text { (In } \\
\left.2 / K_{t}\right)\end{array}$ \\
\hline \multirow{4}{*}{ Low } & Total litter & 21.90 & 7.34 & 2.98 & 0.34 & 0.23 \\
\hline & Leaf litter & 12.88 & 4.13 & 3.12 & 0.32 & 0.22 \\
\hline & Wood litter & 4.10 & 1.59 & 2.58 & 0.39 & 0.27 \\
\hline & "Miscellaneous" litter & 5.86 & 1.67 & 3.51 & 0.29 & 0.20 \\
\hline \multirow{4}{*}{ Mid } & Total litter & 42.85 & 16.08 & 2.67 & 0.38 & 0.26 \\
\hline & Leaf litter & 27.56 & 10.01 & 2.75 & 0.36 & 0.25 \\
\hline & Wood litter & 6.33 & 2.32 & 2.73 & 0.37 & 0.25 \\
\hline & "Miscellaneous" litter & 8.97 & 3.16 & 2.87 & 0.35 & 0.24 \\
\hline \multirow{4}{*}{ High } & Total litter & 47.53 & 16.72 & 2.84 & 0.35 & 0.34 \\
\hline & Leaf litter & 30.91 & 10.71 & 2.89 & 0.35 & 0.24 \\
\hline & Wood litter & 5.58 & 1.72 & 3.24 & 0.31 & 0,21 \\
\hline & "Miscellaneous" litter & 11.04 & 3.76 & 2.94 & 0.34 & 0.23 \\
\hline \multirow{4}{*}{ Mean } & Total litter & 37.43 & 13.38 & 2.80 & 0.36 & 0.25 \\
\hline & Leaf litter & 22.78 & 8.29 & 2.75 & 0.36 & 0.25 \\
\hline & Wood litter & 5.34 & 1.87 & 2.86 & 0.35 & 0.24 \\
\hline & "Miscellaneous" litter & 8.62 & 2.87 & 3.00 & 0.33 & 0.23 \\
\hline
\end{tabular}

$\mathrm{K}_{\mathrm{t}}=$ Turnover rates calculated as litter fall relative to litter biomass by Nye, [25].

$\mathrm{T}_{50}=$ Time required for the composition of half the initial material Edu, [26]. 


\section{Discussion}

\subsection{Leaf Decomposition}

The results of this study show that decomposition rate was species dependent and varied across tidal gradients. The average decomposition rates and the decomposition half lives $\left(\mathrm{T}_{50}\right)$ of the mangrove leaves was at variance with those reported for other mangrove species of the same genera in other regions. The mean decomposition half time for A. africana in this study was 57 days compared to 56 days for temperate A. marina in Sydney [27] and 14 days in Golf St. Vincent, South Australia [28]; for R. racemosa, it was 86 days compared to 43 days for $R$. apiculata and 34 days for R. mucronata in Malaysia [29] and 289 days for $R$. apiculata and 347 days for $R$. mucronata in India [30]. Higher rates of leaf decomposition were observed in macro tidal riverine forests [31]. Decay coefficients vary in different mangrove species and decay rates vary with temperature and geographical region [32]. The results obtained in this study only indicate the possible decomposition rates of the mangrove species in the field. It is possible that the leaves inside decomposition bags as used in this study may face different micro-habitat and micro-climatic conditions than naturally decomposing leaves [33]. Thus the litter bag method used in this experiment is only an estimation of decomposition rates to reflect trends and allow comparison among species and sites.

Table 4. Estimate of projected mean leave litter turnover across tidal gradients (low, mid, high) in a mixed mangal forest (N. fruticans, $R$. racemosa and A. africana) at Esuk Mba of the Great Kwa River of Cross River estuary, Nigeria.

\begin{tabular}{ccccc}
\hline $\begin{array}{c}\text { Tidal } \\
\text { level }\end{array}$ & Species & $\begin{array}{c}\text { Turnover } \\
\text { rate }\left(\mathbf{K}_{\mathbf{d}}\right)(\mathbf{g} \\
\left.\mathbf{d}^{-1} \mathbf{d w t}\right)\end{array}$ & $\begin{array}{c}\text { Residence } \\
\text { time (days) } \\
\left(1 / K_{d}\right)\end{array}$ & $\left.\begin{array}{c}\text { Half life } \\
\left(\mathbf{T}_{\mathbf{5 0}}\right) \\
(\mathbf{I n} 2\end{array} K_{d}\right)$ \\
\hline Low & $\mathrm{Ny}$ & 0.008 & 125.00 & 86.6 \\
& $\mathrm{Rh}$ & 0.010 & 100.00 & 69.3 \\
Mean & $\mathrm{Av}$ & 0.015 & 66.67 & 46.2 \\
Mid & $\mathrm{Ny}$ & 0.011 & 90.91 & 63.01 \\
& $\mathrm{Rh}$ & 0.007 & 142.86 & 99.0 \\
Mean & $\mathrm{Av}$ & 0.012 & 125.00 & 86.6 \\
High & & 0.009 & 111.11 & 77.01 \\
& $\mathrm{Ny}$ & 0.006 & 166.67 & 115.5 \\
& $\mathrm{Rh}$ & 0.006 & 166.67 & 115.5 \\
Mean & $\mathrm{Av}$ & 0.009 & 111.11 & 77.0 \\
Average & $\mathrm{Ny}$ & 0.007 & 142.86 & 99.0 \\
& $\mathrm{Rh}$ & 0.008 & 142.86 & 99.0 \\
& $\mathrm{Av}$ & 0.012 & 83.33 & 86.6 \\
\hline
\end{tabular}

$\mathrm{K}=$ Projected turnover rates calculated as mean leaf decay coefficient Mindaman, [22]

$\mathrm{T}_{50}=$ Time required for decomposition of half the initial material Species: $\mathrm{Av}=$ Avicennia africana, $\mathrm{Ny}=$ Nypa fruticans, $\mathrm{Rh}=$ Rhizophora racemosa
Table 5. Comparison of leaf litter turnover based on projected mean decomposition rate $\left(K_{d}\right)$ and actual turnover rates based on relative measure of leaf litter fall and leaf litter on the forest floor $\left(K_{t}\right)$ across tidal gradients (low, mid, high) in mixed mangal forest ( $N$. fruticans, $R$. racemosa and A. af ricana) at Esuk Mba of the Great Kwa River of Cross River estuary, Nigeria.

\begin{tabular}{|c|c|c|c|c|c|c|}
\hline \multirow[t]{2}{*}{$\begin{array}{l}\text { Tidal } \\
\text { level }\end{array}$} & \multicolumn{2}{|c|}{$\begin{array}{c}\text { Turnover rates } \\
(\mathbf{K}) \\
\left(\mathrm{g} \mathrm{d}^{-1}, \mathbf{d w t}\right)\end{array}$} & \multicolumn{2}{|c|}{$\begin{array}{l}\text { Residence time } \\
\text { ( } 1 / \mathrm{K}) \text { (days) }\end{array}$} & \multicolumn{2}{|c|}{$\begin{array}{l}\text { Half life }\left(\mathbf{T}_{50}\right) \\
\quad \text { (days) }\end{array}$} \\
\hline & $\mathbf{K}_{\mathbf{d}}$ & $\mathbf{K}_{\mathrm{t}}$ & $1 / K_{d}$ & $1 / K_{t}$ & $2 / K_{d}$ & $2 / K$ \\
\hline Low & 0.011 & 3.12 & 90.91 & 0.33 & 63.01 & 0.22 \\
\hline Mid & 0.009 & 2.75 & 111.11 & 0.36 & 77.02 & 0.25 \\
\hline High & 0.007 & 2.89 & 142.86 & 0.35 & 99.02 & 0.24 \\
\hline
\end{tabular}

$\mathrm{K}_{\mathrm{t}}=$ Turnover rates calculated as litter fall relative to litter biomass [25, 26] $\mathrm{K}_{\mathrm{d}}=$ Projected turnover rates calculated as mean leaf decay coefficient [22]

$\mathrm{T}_{50}=$ Time required for decomposition

Leaf decomposition rates among mangrove wetlands have been linked to differences in leaf quality (morphology, texture and composition), especially relating to two key factors: the tannin concentration and nutritional value [31]. High tannin concentrations are aversive to detritivores and inhibit microbial activity [34], while nutritional value refers to the $\mathrm{C}: \mathrm{N}$ ratio where low ratio indicates higher nitrogen concentration and higher nutritional quality. From the results obtained $A$. africana had the lowest and $R$. racemosa the highest tannin concentration and also C:N ratio. The morphological quality of $A$. africana may be accountable for its fast rate of decomposition compared to the other species studied. Such qualities may include thin leaves and their ability to fragment and sink fast, leading to an easy onset of decomposition. In contrast, $R$. racemosa and $N$. fruticans have thick leaves and are buoyant, floating for several days before sinking, thus increasing their chances of being washed out into the estuary [29].

Decomposition rates have been found to be site and species dependent. They are affected by tidal inundation frequency, air and water temperature, chemical and nutritional contents of leaf material and decomposer species composition [29, 35]. Investigations reveal that leaf litter decomposition rates are higher in low intertidal regions which are inundated by tides daily than in high intertidal regions inundated by tides less frequently [4]. The degree of submersion of leaves and twigs influences speed of leaching and hence affects decomposition rates. The rapid decomposition rate is attributed to the rapid leaching of tannins from submerged leaves and greater stability and predictability of environmental conditions, with bacterial and fungal colonization being much more rapid under such conditions. Leaf litter decomposition rates have been found to be higher in forests at lower latitudes. This has been attributed to the influence of air and water temperatures on decomposition rates [36]. Differences in species significantly affect leaf decomposition rates. This is attributed to differences in biochemical complexity and 
elemental concentrations particularly nitrogen. Leaves of Avicennia have high initial nitrogen concentration, low C: $\mathrm{N}$ ratio and low concentration of tannins compared to Rhizophora and Nypa with high tannin concentration, high $\mathrm{C}$ : $\mathrm{N}$ ratio and relatively impervious cuticle. Hence, decomposition rate is faster in Avicennia. Variations in relative decomposition rates of mangrove tissues within and among mangrove forests which may be attributable to differences in biotic and abiotic factors play important roles in retention and recycling of organic matter which is important in vertical accretion and habitat stability $[37,38$, 4].

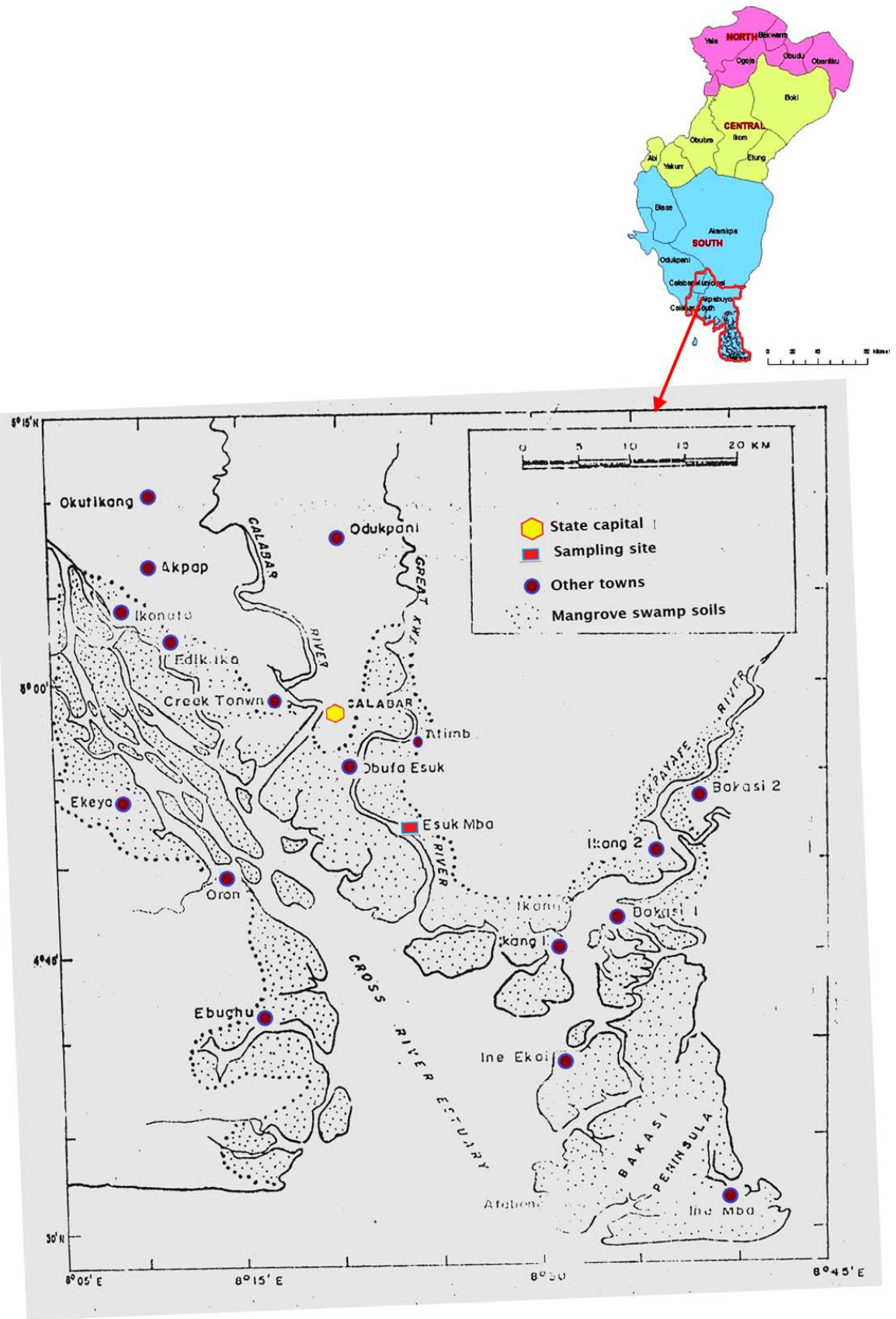

Fig. 1. Map of Cross River estuary

Microorganisms (detritivores) play vital roles in accelerating litter decomposition and their relative influence on organic matter degradation varies spatially within mangrove forests. Microbial decay of organic material is the main process for the release of soil nitrogen under the direct influence of temperature and moisture [39]. Variability in leaf litter decomposition between tidal levels may be influenced by tidal inundation with increased submergence accelerating decomposition at the low tide level. [40, 41]

The low tide level exposes the decomposing leaves to constant water soaking which causes leaching of labile materials and promotes microbial activity. The physical impact of tides on the leaves may also cause fragmentation 
and increase weight loss [13]. However, the higher rates of decomposition observed at the high tide level where there is less frequent tidal inundation may be attributed to the high density of microbial populations as observed in previous studies [42, 43]. Overall, tidal level had a greater effect on decomposition rate than species suggesting that external factors may contribute more in determining the decomposition rate in this study. The results of this study support numerous studies in tropical mangrove forests on litter dynamics and its contribution to nutrient cycles and support of food webs in coastal habitats [44, 45, 41]. The present study has implication for understanding and predicting patterns of accumulation and distribution of organic matter in Nigerian mangal ecosystem. Understanding the mechanisms controlling organic matter provides insight into carbon storage potential [46] and biogeochemical cycles of nutrients [47].The low decomposition rates recorded in this study may imply a maximal export of litter from this mangrove system [30]. The results of the leaf turnover rates indicate that the actual residence times of leaf litter across the tidal gradient were much shorter than the projected time based on leaf decomposition rates. Based on the wide differences in leaf turnover rates, it can be assumed that geophysical and ecological processes, such as tides of riverine mangroves result in litter turnover rates higher than those expected on the basis of leaf decomposition. This leaf litter turnover supports the hypothesis that geographical factors may be more significant in influencing the fate of leaf litter on the forest floor by exporting them to near shore waters to fuel productivity of the seas especially the nearby estuaries [48]. The decomposition, amount and fate of litter in this mixed mangrove forests in Cross River State, Nigeria are comparable to mixed mangrove forests in other tropical locations.

\subsection{Leaf Litter and Nutrient Dynamics}

The actual leaf litter turnover rate $\mathrm{K}_{\mathrm{t}}$, based on the relative measure of leaf fall and leaf litter on the forest floor, integrates all the ecological processes that enhance leaf loss from the forest floor, including tidal export, decomposition and natural consumption by detritivores and macro consumers. The projected leaf decomposition rates $\mathrm{K}_{d}$ indicate leaf loss without the influence of tidal export or consumption by macro consumers (which are excluded by mesh bags for decomposition). The difference observed between these two coefficients of leaf litter turnover indicates the relative contribution of other ecological processes to leaf litter loss. That is, the difference in $\mathrm{K}_{\mathrm{t}}$ and $\mathrm{K}_{\mathrm{d}}$ of leaf litter turnover in this study suggest the existence of mechanisms of litter loss other than leaf decomposition. Since this mangrove forest is inundated frequently by tides and the actual residence times of leaf litter across the tidal gradients were much shorter than the projected time based on leaf decomposition rates, it is most likely that most of the leaf fall will be transported to the Cross River estuary.

The average amount of litter biomass on the forest floor generally decreased during the rainy season (June September) especially at the low tide level [26]. This decrease may be associated with greater export, due to increased tidal effects during the rainy season on the transport of litter from the forest floor [49]. Nypa and Rhizphora leaves which dominate the low tide level have bouyance and floating ability (due to their biological quality) increasing the chance of their being washed out into the estuary. These have implications for organic matter dynamics and fisheries in the estuary. The rate at which decomposition takes place at the forest floor controls how much organic matter is recycled within the forest and how much is exported to near-shore waters [33]. Higher rates of decomposition result in nutrient retention while slower rates increase the chance of leaves being exported [50]. Accumulation of nitrogen through time is responsible for the decrease in $\mathrm{C}: \mathrm{N}$ ratios in the decomposing leaves used in the decomposition study. The ratios decreased from about 27 for $A$. africana, 36 for $R$. racemosa and 32 for $N$. fruticans at the beginning of the study to 22.7, 23.9 and 23.6 respectively, after 56 days. Nitrogen enrichment and subsequent decrease in $\mathrm{C}: \mathrm{N}$ ratio of decomposing litter has been indicated in other studies in mangrove litter decomposition $[30,51]$. This enrichment may be caused by action of bacterial and fungal colonization of the decomposing leaves [52]. The differences in nitrogen accumulation through time in the different species and at different tidal levels may be as a result of differences in the lignin content of the leaves and the aerobics at the tidal levels. Lignin decomposition is an oxidative process [53]. It may be that lignin decomposing fungi are absent or cannot function properly in mid and high tidal levels due to their low oxygen and aneroxic conditions contributing to the much slower rate of decomposition and lower nitrogen accumulation rate in Nypa and Rhizophora leaves which have high lignin content [54]. Leaves subjected to frequent oxygenation and wetting by tidal inundation have enhanced decomposition [40, 4, 41]. The difference in rates of accumulation of nitrogen in the decomposing leaves across the tidal levels may also be attributable to the soil characteristics of these tidal levels. It has been observed that litter decomposing in forest sites with higher soil nitrogen concentration has higher rates of accumulation of nitrogen [55]. Similar study in the area showed that the high tide level was observed to be characterised by higher soil nitrogen concentration relative to other tidal levels [42, 56, 57]. The increase of nitrogen concentration and nutritional content of mangrove leaves during decomposition may have been mediated by microbial and fungal populations which colonize and carry out saprophytic decomposition. The increase was significantly high $(\mathrm{P}<0.001)$ at the low tide level. Submersion in water results in faster rate of microbial colonization and subsequent decomposition due to the physical impact of tides and leaching on the leaves [33]. The faster rate of decomposing litter leads to probable more accumulation of nitrogen biomass on litter. The nitrogen concentration and 
nutritional contents of the mangrove leaf litter increased during decomposition. There were species differences in nitrogen accumulation through time and across tidal levels. These differences may be due to species-specific differences in leaf composition, oxygen availability, soil characteristics and feeding activity of micro organisms [49]. The relative contribution of decomposition to litter dynamics and nutrient recycling varies along a tidal gradient. As tidal inundation frequency decreases, the litter dynamics become more influenced by the decomposition and respiratory characteristics of the forest floor mediated by microbial and fungal populations which carry out saprophytic decomposition. This increases the nitrogen enrichment of the decomposing litter. Decrease in C:N ratios is also indicated in other studies on litter decomposition [31]. Calculations of net changes in nutrients (immobilization / mineralization) revealed differences among species and across the tidal gradient. The average over species and tidal level showed a net mineralization of nutrients (nitrogen and carbon) in all the decomposing litter, implying that the nutrients in the decomposing litter are mineralized, further supporting productivity in the system [50].

The trophic value and organic matter exchange between mangroves and adjacent ecosystems is a subject of ongoing debate and research [58]. The long standing ecological paradigm that mangrove detritus formed the foundation of a decomposer food web in mangrove estuaries and also exported offshore to contribute to coastal secondary production [59] has been questioned. Studies using stable carbon isotope ratios reveal that although mangrove detritus play significant trophic role in the ecosystem and the coastal zone, its contribution to nutrition of higher trophic levels decreases rapidly away from the mangrove zone $[60,61,62]$. In estuaries with high proportion of mangrove forest to open water, mangrove detritus is thought to be significant in trophic value to consumers within the mangrove habitat [8]. Mangrove based detritus play vital roles in secondary productivity of adjacent water bodies. The microorganisms which colonize leaf litter during decomposition also serve as food source for higher trophic level consumers [8]. Since mangroves are the dominant primary producers in this system and bacterial activity under the mangroves is very high [63] reflecting relatively high rate of organic input, it can be concluded that energy in this system is largely derived from mangrove litter and channeled through benthic decomposer food chain. The process of decomposition of mangrove litter which leads to derivation of detritus and subsequent release of nutrients is important in nutrient dynamics within the mangrove forest as well as in offshore systems [59, 16].

\section{Conclusion}

The results of this study have implications in the understanding and prediction of the dynamic functions and processes of the mangrove ecosystem in the Cross River estuary. Understanding the nature of nutrient conditions and transfers in coastal forests is important for forest renewal and long term sustainability of forest resources. Knowledge of the nature of the processes of nutrient exchanges and transfers provide insight into nutrient dynamics, biogeochemical cycles and energy flow which are critical for the maintenance and long term sustainability of the forests and other ecological resources. Comparatively little is known about nutrient transfers among different forest types and forest ecosystems to adjacent marine waters in Nigeria - whether they are "source" or 'sink' environments. This is the first study of litter dynamics in a riverine mangrove system of the Cross River estuary. Further research is required to establish the relevance of exported particulate and dissolved organic matter from the mangrove forests to fuel productivity of the adjacent marine waters especially for the Cross River estuary and the Gulf of Guinea. The mangrove forests of the Great Kwa River in the Cross River estuary may be considered to function as nutrient source to the environments. However, sufficient studies on other possible sources are carried out, this assumption of the trophic importance of mangrove detritus relative to other carbon sources in Cross River estuary remains ambiguous. In order to fully understand the nutrient dynamics of a system, the following aspects are essential: budgets of inputs and losses, sources of nutrients and their cycling in ecosystems, criteria and indicators to effectively monitor the ecological state of the ecosystems under different regimes of forest use and management. There is dearth of information on these aspects on the coastal forests of Nigeria and the Cross River estuary. Understanding the nature of nutrient conditions and transfers in coastal forests is important for forest renewal and long-term sustainability of forest resources. Further studies on these aspects are essential to enhance better understanding of the processes and functioning of mangrove and surrounding ecosystems. Such understanding will aid in the development of management plans to protect our mangroves.

\section{References}

[1] Alongi, D. M. Present state and future of the world's mangrove forests. Environmental Conservation, Vol. 29, pp.231-349. 2002.

[2] World Bank, International Society for Mangrove Ecosystems, Center Aarhus, Principles for a code of conduct for the management and sustainable use of mangrove ecosystems. The World Bank, Washington, D.C. 211 pp. 2004.

[3] Hogarth, P. J. The Biology of Mangroves. Oxford University Press. 272pp. 1999.

[4] Middleton, B. A. and McKee, K. L. Degradation of mangrove tissues and implications for peat formation in Belizean Island forest. Journal of Ecology, Vol. 89, pp.818828. 2001. 
[5] Ellison, A. M. and Farnsworth, E. J. Mangrove communities In: Bertness, M. D., Gaines, S. D. and Hay, M. E. (eds). Mangrove Community Ecology, (432 - 442). Sinauer Associates, Sunderland, Massachetts, U. S. A. 2001.

[6] Rey, J. R. and Rutledge, C. R. Mangroves. http://edis.ifas.ufi.edu/in195. 2005. Retrieved 16/02/06.

[7] Ong, J. E. The ecology of mangrove conservation and management. Hydrobiologia, Vol. 295, pp.343-351. 1995.

[8] McKee, K. L. and Faulkner, P. L. Restoration of biogeochemical function in mangrove forests. Restoration Ecology, Vol. 8, pp. 247-259. 2000.

[9] Lee, S. Y. Mangrove outwelling: A review. Hydrobiologia, Vol. 295, pp.203-212. 1995.

[10] Sunil-Kumar, R. A review of biodiversity studies of soil dwelling organisms in Indian mangroves Zoos Print Journal, Vol.15 No.3, pp. 221-227. 2000.

[11] Sunil-Kumar, R. Mangrove debritus loading, tidal export and its large scale effect on promoting biodiversity resources. http://chennai.sancharnet.in/d/envasscas/9(13).htm. Retrieved 02/06/05. 2005.

[12] Wardle, D. A. Bonner, K. I. and Nicholson, K. S. Biodiversity and plant litter: experimental evidence which does not support the view that enhanced species richness improves ecosystem function. Oikos, Vol. 79, pp. 247-258. 1997.

[13] Feller, I. C., Whigham, D. F., O’Neill, J. P. and McKee, K. L. Effects of nutrient enrichment on within stand cycling in a mangrove forest. Ecology, Vol. 8,pp. 2193-2205. 1999.

[14] Alongi, D. M. Mangrove - microbe - soil relations. In: Kristensen, E., Haesse, R. R., Kostka, J. E. (Eds), Interactions between macro and micro-organisms in marine sediments, (85-103). Coastal and estuarine studies, volume 60 American Geophysical Union, Washington DC. 2005.

[15] Alongi, D. M., Clough, B. F. and Robertson, A. J. Nutrientuse efficiency in arid-zone forests of the mangrove Rhizophora stylosa and Avicennia marina. Aquatic Botany, Vol, 82, pp.121-131. 2005.

[16] Dittmar, T., Hertkorm, N., Kattner, G. and Lara, R. J. Mangroves, a major source of dissolved organic carbon to the oceans. Global Biogeochemical Cycles, 20, GB 1012. doi:1029/2005GB0025. 2006.

[17] Akpan, E. R. Influence of meteorological and hydrographic factors on the water quality of the Calabar river, Nigeria. Tropical Journal of Environmental Research, Vol.2, No.182, pp.107-111. 2000.

[18] Holzloehner, S., Nwosu, F. M. and Akpan, E. R. Mangrove mapping in the Cross River estuary, Nigeria. African Journal of Environmental Pollution and Health, Vol. 1, No.2, pp. 76-87. 2002.

[19] NMOC. Meteeorological data for 2008/2009. Nigeria meteorological office, Margaret Ekpo International Airport, Calabar. 2010

[20] Fell, I. W. and Master, I. M. Litter decomposition and nutrient enrichments. In: Snedaker, S. C. and Snedaker, J. G. (Eds.). The Mangrove Ecosystem Research Method, (239263). UNESCO, United Kingdom. 1984.
[21] APHA, Standard methods for the examination of water and waste water, Washington DC. PHHA-AWWA-WPCF, 1134 pp. 1980.

[22] Minderman, G. Addition, decomposition and accumulation of organic matter in forest. Journal of Ecology, Vol.56, pp.335-362. 1968.

[23] Obi, J. U. Statistical methods of determining differences between treatment means and research methodology issues in laboratory and field experiments. Enugu: SNAAP Press. 717pp. 2002.

[24] Harmon, M. E., Franklin, J. F., Swanson, F. J., Sollins, P., Gregory, S. V., Lattin, J. P., Anderson, N. H., Cline, S. A., Aumen, N. G., Sedell, J. R., Lienkaemper, G. W., Gromack, K. J. and Cummins, K. W. Ecology of coarse woody debris in temperate ecosystems. Advances in Ecological Research, Vol.15, pp.133 - 302. 1986.

[25] Nye, P. H. Organic matter and nutrient cycles under moist tropical forests. Plant Soil, Vol.13, pp.333 - 346. 1961.

[26] Edu, E. A. B. Litter dynamics (Production, Composition and Decomposition) of mangroves in a mixed riverine mangrove forest of the Cross River estuary, Nigeria. Ph.D Thesis, University of Calabar, Calabar, Nigeria. 2012.

[27] Goulter, P. F. E. and Allaway, W. G. Litter fall and decomposition in a mangrove stand Avecennia marina (Forsk) Vieth. Australian Journal of Marine and Freshwater Resources, Middle Harbour, Sydney, Vol.30, pp.541 - 546. 1979.

[28] Imgraben, S. and Dittmann, S. Leaf litter dynamics and litter consumption in two temperate South Australian mangrove forests. Journal of Sea Research, Vol.59, pp.83-93. 2008.

[29] Ashton, E. C., Hogarth, P. J. and Ormond, R. Breakdown of mangrove leaf litter in a managed mangrove forest in Peninsular Malaysia. Hydrobiologia, Vol.413, pp.77-88. 1999.

[30] Wafar, S., Untawale, A. G. and Wafar, M. Litterfall and energy flux in a mangrove ecosystem. Estuarine, Coastal and Shelf Science, Vol.44, pp.111-124. 1997.

[31] Twilley, R. R., Pozo, M., Garcia, V. H., Rivera-Monroy, V. H., Zambrano, R. and Bodero, A. Litter dynamics in riverine mangrove forests in the Guayas River Estuary, Ecuador.Oecologia, Vol.111, pp.109-122. 1997.

[32] Tam, M. F. Y., Li, S. H., Lan, C. Y., Chen, G. Z., Li, M. S. and Wong, Y. S. 1998. Nutrients and heavy metal contamination of plants and sediments in Futian mangrove forest. Hydrobiologia, Vol.295, No.1 - 3, pp.149 - 158.

[33] Boulton, A. J. and Boon, P. I. A review of methodology used to measure leaf litter decomposition in lotic environments: time to turn over an old leaf? Australian Journal of Marine Freshwater Resources, Vol.42, pp.1 - 43. 1991.

[34] Steinke, T. D., Holland, A. J. and Singh, Y. Leachira losses during decomposition of mangrove leaf litter. South African Journal of Botany, Vol.59, pp.21-25. 1992.

[35] Bosire, J. D., Dahdouh-Guebas, F., Kairo, S. G., Kazungu, J., Dehairs, F. and Koedam, N. Litter degradation and CN dynamics in reforested mangrove plantations at Gazi Bay, Kenya. Biological Conservation, Vol.126, pp.287-295. 2005. 
[36] Cattiano, J. H., Anderson, A. B., Rombold, J. S.and Nlepstad, D. C. Phenology, litterfall, growth and root biomass in a tidal flood plain forest in the Amazon estuary. Revista Brasileira de Botanica, Vol.25, pp.419 - 430. 2004.

[37] Lee, S. Y. Ecological role of graspid crabs in mangrove ecosystems: a review. Marine and Freshwater Resources, Vol.49, pp.335 - 343. 1998.

[38] Alongi, D. M. Coastal Ecosystem Processes. Boca Raton. CRC Press, 419pp. 1998.

[39] Smithwick, E. A. H., Turnei, M. G., Mack, M. C. G., Chapin III, F. S., Shu, Jun and Balser, T. C. Spatial heterogeneity in ecosystem processes after severe fire in a black spruce $(P$. mariana) forest: Alaska (USA). Ecological Society of America 89th Annual Meeting, (August 1-6) Portland Oregon. 314pp. 2004.

[40] Dick, T. M. and Osunkoya, O. O. Influence of tidal restriction floodgates on decomposition of mangrove litter. Aquatic Botany, Vol.68, pp. 273-280. 2000.

[41] Mfilinge, P. L., Atta, N. and Tsuchiya, M. Nutrient dynamics and leaf litter decomposition in a subtropical mangrove forest at Oura Bay, Okinawa, Japan. Trees, Vol.6, pp.172180. 2002.

[42] Edu, E. A. B. Microflora populations in riverine mangrove sediments, Cross River estuary, Nigeria Masters Thesis. University of Calabar, Calabar, Nigeria 92pp. 2005.

[43] Edu, E. A. B., Omokaro, D. N., Holzloehner, S. and Udensi U. Microflora populations in mangrove sediments of Cross River estuary. Global Journal of Pure and Applied Sciences, Vol.13, No.3, pp.347-352. 2007.

[44] Holmer, M. and Olsen, A. B. Role of decomposition of mangrove and seagrass detritus in sediment carbon and nitrogen cycling in a tropical mangrove forest. Marine Ecological Progress Series, Vol.230, pp.87-101. 2002.

[45] Jennerjahn, T. C. and Ittekkot, V. Relevance of mangroves for the production and deposition of organic matter along tropical coastlines. Naturwissenschaften, Vol.89, 23-30. 2002.

[46] Fujimoto, K., Imaya, A., Tabuchi, R., Kuramoto, S., Utsugi, $\mathrm{H}$. and Murofushi, T. Blowground carbon storage of Micronessian mangrove forests. Ecological Research, Vol.14, pp.409 - 413. 1999.

[47] Chen, R.R. and Twilley, R. A simulation model of organic matter and nutrient accumulation in mangrove wetland soils. Biogeochemistry, Vol.44, pp.93-118. 1999.

[48] Van der Valk, A. G. and Attiwill, P. M. Decomposition of leaf and root litter of Avicennia marina at Westernport Bay, Victoria, Australia. Aquatic Botany, Vol.18, pp.205-221. 1984.

[49] Twilley, R. R., Lugo, A. F. and Patterson - Zucca, C. Production, standing crop and decomposition of litter in basin mangrove forests in southwest Florida. Ecology, Vol.67, pp.670 - 683. 1986.
[50] Werry, J. and Lee, S. Y. Graspid crabs mediate link between mangrove litter production and planktonic food chains. Marine Ecology Progress Series, Vol.293, pp.165-176. 2005.

[51] Woitchik, A. F., Ohowa, B., Kazungu, J. M., Rao, R. G., Goeyens, I. and Dehairs, F. Nitrogen enrichment during decomposition of mangrove litter in an east African coastal lagoon (Kenya): relative importance of biological nitrogen fixation. Biogeochemistry, Vol.39, pp.15-35. 1997.

[52] Lee, S. Y. Potential trophic importance of the faecal material of the mangrove crab Sesarma messa. Marine Ecology Progress Series, Vol.158, pp.275 - 284. 1997.

[53] Reid, I. D. Biodegradatio of lignin. Canadian Journal of Botany, Vol.73, No.1, pp.51011 - 51018. 1995.

[54] Sjoberg, G. Lignin degradation: Long term effects of nitrogen addition on decomposition of forest soil organic matter. Doctoral Thesis. Swedish University of Agricultural Sciences, Uppsala, Sweden 46pp. 2003.

[55] Romero, L. M., Smith III, I. J. and Fourqurean, J. W. Changes in mass and nutrient content of wood during decomposition in a South Florida mangrove forest Journal of Ecology, Vol.93, pp.618-631. 2005.

[56] Edu, E. A., Omokaro, D. N. and Nya, P. J. Influence of forest types on the physicochemical parameters in mangrove sediments of Cross River estuary, Nigeria. Nigerian Journal of Botany, Vol.21, No.1,pp. 179-186. 2008a.

[57] Edu, E. A., Omokaro, D. N. and Nya, P. J. Microflora and nutrient status in the mangrove sediments of Cross River estuary, Nigeria. Nigerian Journal of Experimental and Applied Biology, Vol.9, No.2, pp.109-118. 2008b.

[58] Bouillon, S., Connolly, R. M. and Lee, S. Y. Organic matter exchange and cycling in mangrove ecosystems: recent insights from stable isotope studies. Journal of Sea Research, Vol.59, pp.44-58. 2008.

[59] Dittmar, T., Lara, R. J. and Kattner, G. River or mangrove? Tracing major organic matter sources in tropical Brazilian coastal waters. Marine Chemistry, Vol.73, pp.253-271. 2001.

[60] Lee, S. Y. Carbon dynamics of Deep Bay eastern Pearl River estuary, Chinall: Trophic relationship based on carbon and nitrogen stable isotopes. Marine Ecology Progress Series, Vol.205, pp.51-70. 2000.

[61] Lee, S. Y. Mangrove macrobenthos. Assemblages, services and linkages. Journal of Sea Research, Vol.59, pp.16-29. 2008 .

[62] Macia, A. Primary carbon sources for juvenile penaeid shrimps in a mangrove-fringed Bay of Inhaca island, Mozambique: A dual carbon and nitrogen isotope analysis. West Indian Ocean Journal of Marine Science, Vol.3, pp.151-161. 2004.

[63] Panikov, N. S. Understanding and prediction of soil microbial community dynamics under global change. International Journal of Applied Soil Ecology, Vol.5, No.1,pp. 1 - 17. 1999. 